\title{
6.4 Interaction Between the Sleep-Wake Cycle and the Rhythm of Rectal Temperature
}

\author{
J. Zulley and R.A. Wever ${ }^{1}$
}

\section{Introduction}

The human circadian system has been considered as controlled by two - or even more oscillators which are represented by the sleep-wake cycle and the rhythm of rectal temperature (Wever 1975). Both rhythms are influenced by environmental time cues but also affect each other mutually. The interaction between the two rhythms is especially obvious in the absence of external stimuli, i.e., under constant conditions, where it usually results in mutual synchronization between the two rhythms. However, in about $30 \%$ of subjects examined under constant conditions, the two rhythms show different average periods in the steady state; nevertheless, they continue to interact mutually. It is only in this state of internal desynchronization that the direction of the interaction between the two rhythms can be evaluated.

\section{Method}

Human subjects lived in complete isolation from environmental time cues and, hence, showed free-running circadian rhythms. The illumination in the experimental room was constant in some experiments and self-selected (i.e., light during wakefulness and dark during sleep) in others. Beside a variety of physiological and psychological variables, the state of activity and rectal temperature was recorded continuously (Wever 1979). In some of these studies, sleep was recorded polygraphically by measuring EEG, EOG, and EMG (Zulley 1979). In all experiments under consideration, the subjects lived singly isolated for, at least, 4 weeks.

Out of the larger sample of data, free-running rhythms were analyzed from ten subjects, who all showed internal desynchronization with considerably lengthened sleep-wake cycles. The temporal relationship between the rhythms of activity and rectal temperature (temporal positions of sleep episodes and of minimum values of rectal temperature), and the range of the temperature rhythm (amplitude) will be considered here. In this paper, the term "sleep" is throughout used synonymous to "bedrest" (cf. Glossary in Sleep, 2:287-288, 1980); in the two out of the ten subjects where sleep was recorded polygraphically, the difference between sleep and bedrest was less than half an hour and, hence, negligible with regard to the present investigations.

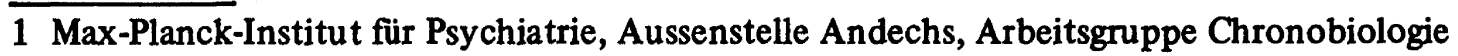




\section{Results}

To illustrate the phenomenon of internal desynchronization, Fig. 1 presents an experiment where the rhythms of sleep-wake and rectal temperature took different periods during the whole experiment of 29 days duration. The activity rhythm had a mean period of $30.2 \mathrm{~h}$ and the rhythm of rectal temperature one of $25.0 \mathrm{~h}$. Consequently, the phase relationship between both rhythms varied from cycle to cycle.

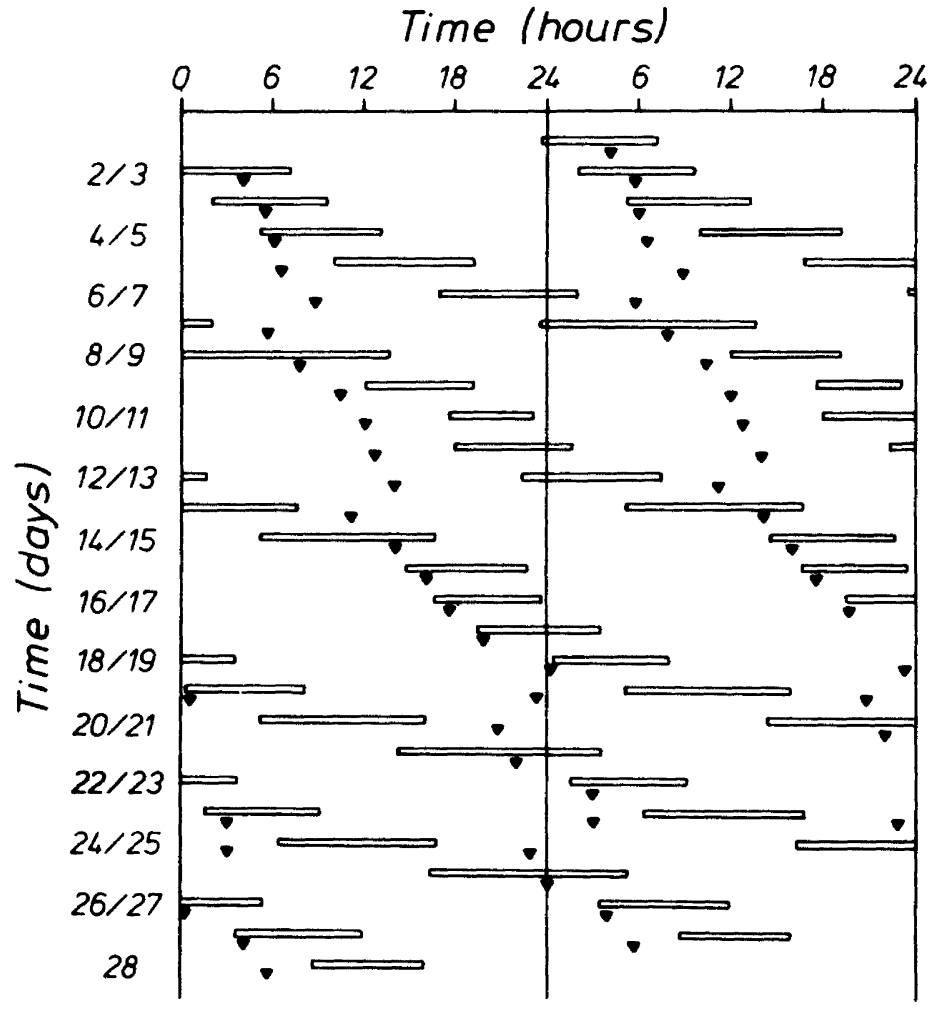

Fig. 1. Consecutive sleep episodes (bars bedrest) and minima of rectal temperature (triangles) of a subject living alone in isolation from environmental time cues. Double plot

For further analyses, the time of every single sleep episodes was recorded with reference to the actual minimum of rectal temperature. Figure 2 shows, in its upper part, the frequency distribution of sleep onsets relative to the temperature minimum. According to the state of internal desynchronization, the phase relationships cover the full cycle. They are, however, not randomly distributed, but show several clearly separated peaks: for the present analysis, the data are divided in two major groups with the centers of gravity of sleep onset at 6.70 and $0.98 \mathrm{~h}$ before the actual temperature minima, and minor groups with the centers of gravity at 4.8 and $11.5 \mathrm{~h}$ after the actual minima (cf. Table 1). The lower part of Fig. 2 shows the dependency of the duration of sleep on its position within the temperature cycle. It demonstrates that longer sleep episodes are typically placed, with their midpoints, before the minimum, and shorter sleep episodes after the minimum of rectal temperature.

A more detailed analysis of this dependency is presented in Fig. 3. It shows the correlation between the duration of the single sleep episodes and the position of sleep within the temperature cycle, separately for onset and end of every sleep episode. As Fig. 3 shows especially for sleep onset, the wave form of this rhythmic relationship is not symmetric, but its ascending slope is much steeper than its descending slope. 


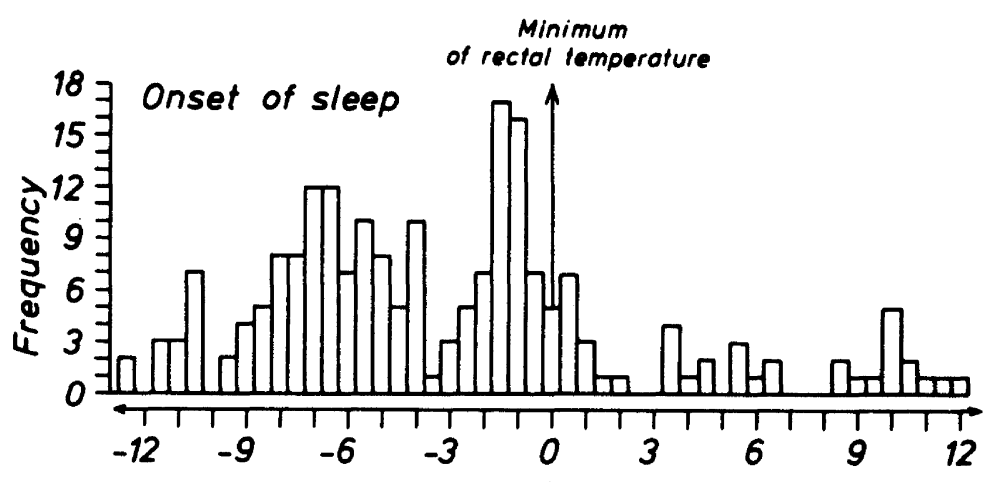

Fig. 2. Upper part Frequency distribution for onset of sleep referred to the time of the actual minimum of rectal temperature. Lower part Duration of sleep correlated to the phase position of the middle of sleep within the temperature cycle (reference: actual minimum of rectal temper-

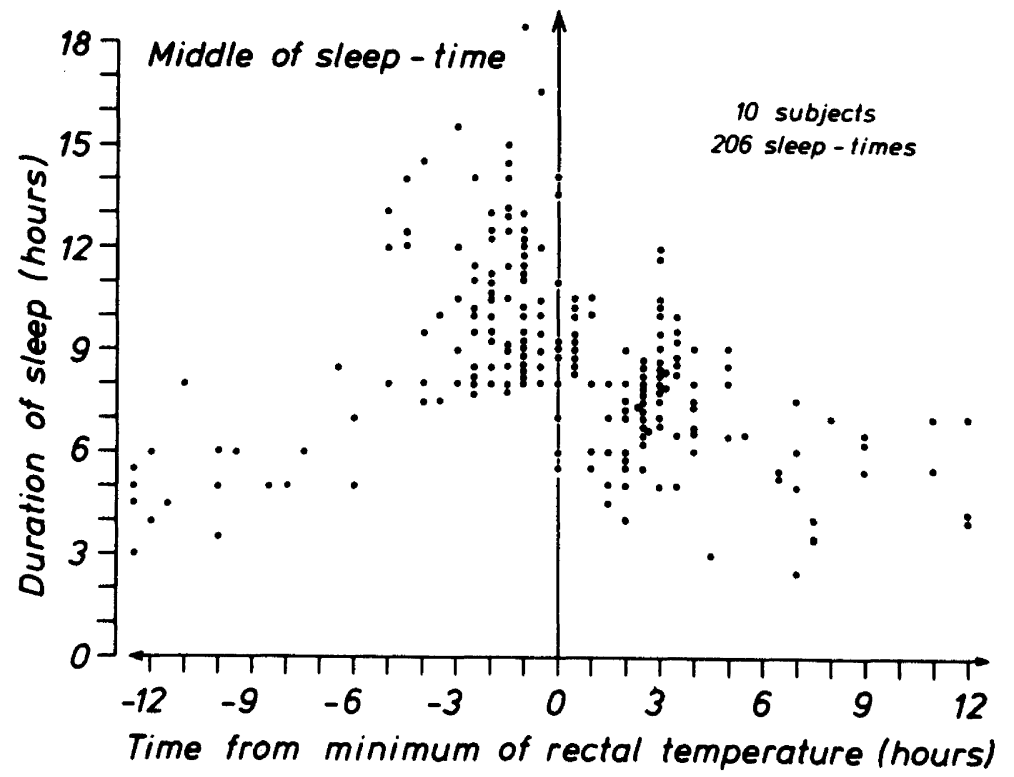
ature)

Around the temperature maximum, there seems even to be an ambiguity with long and short durations of sleep beginning at the same phase. This ambiguity, however, is simulated by the superposition of data from many subjects within one plot. The data from single subjects do not show such an overlap of sleep durations. The meaning is, therefore, that the steep increase in the relationship mentioned occurs in the different subjects at slightly different phases.

In Fig. 3, again, a clustering of the sleep episodes in four groups is recognizable. To elucidate this ordering, the four groups (cf. Fig. 2, upper diagram) are indicated by envelops; average data of all groups are given in Table 1. The comparison of the arrangements of the different groups in the two diagrams concerning onset and end of sleep elucidates the fact that the different groups are recognizable in the distribution of sleep onset but not in that of sleep termination (Zulley et al. 1981): with regard to the phases of sleep onset, for instance, group A is clearly separated from group B; for end of sleep, both groups overlap in their phases so that a monomodal distribution of phases results which is advanced in comparison to that of the sole consideration of group $\mathbf{B}$.

In summary, Fig. 3 demonstrates the dependency of the duration of a sleep episode on its temporal position within the temperature cycle and, hence, the influence of the temperature rhythm on the activity rhythm. Moreover, Fig. 3 demonstrates again the multimodal distribution of mutual phase relationships. Whereas in the state of internal synchronization sleep onset occurs preferably at one certain position with- 


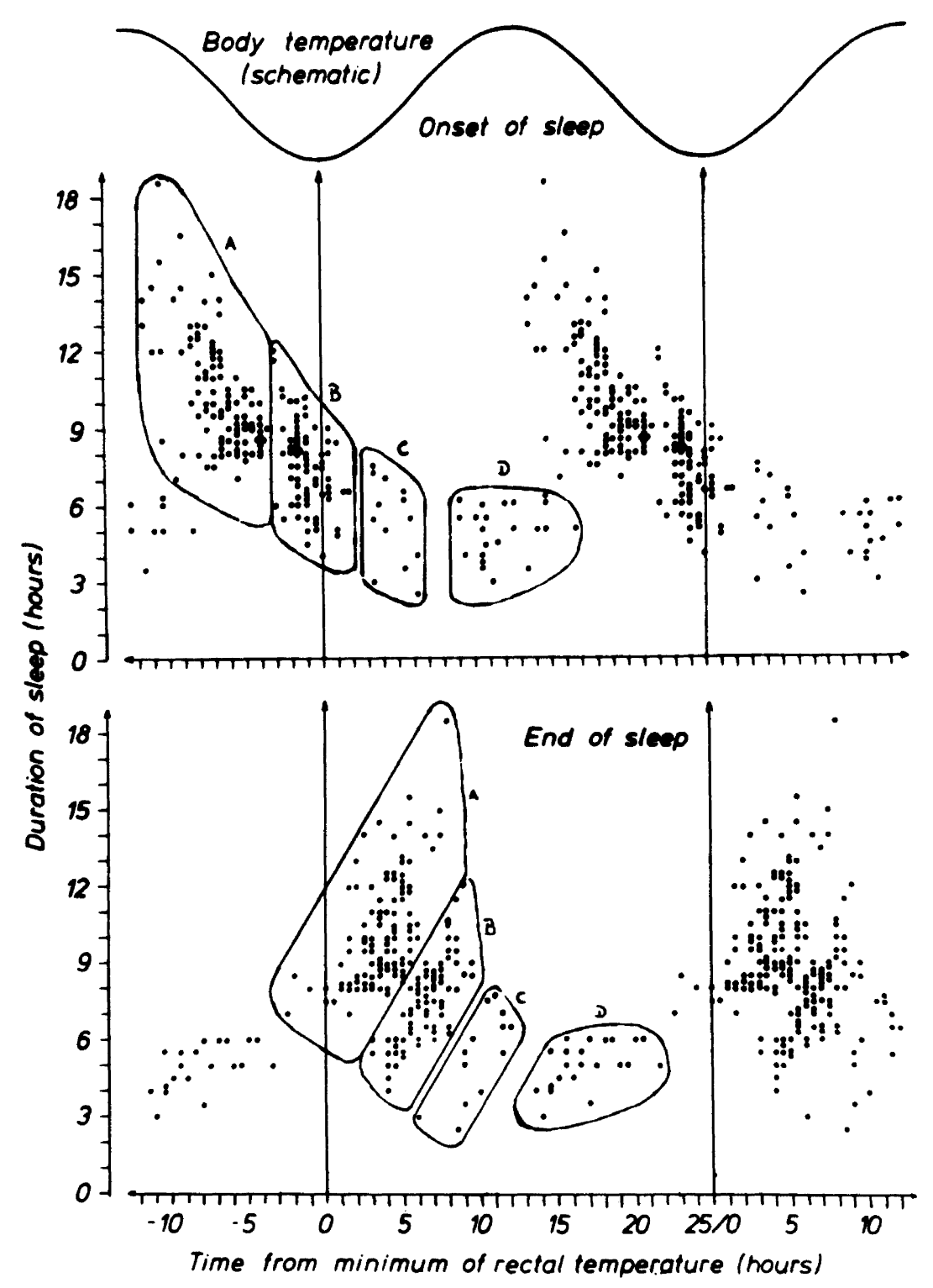

Fig. 3. Duration of sleep correlated to the phase position of sleep onset (above) and end of sleep (below) within the temperature cycle (reference: actual minimum of rectal temperature); double plot. Upper border schematic representation of rectal temperature, for comparison. In the two diagrams corresponding groups of data are indicated each once in the two plots; denotations of the groups corresponds to Table 1

Table 1. Relation between sleep episodes and minima of rectal temperature. (Data from 206 sleep episodes originating from ten subjects)

\begin{tabular}{|c|c|c|c|c|c|}
\hline \multirow[t]{2}{*}{$\begin{array}{l}\text { Group } \\
\text { (cf. Fig. 3) }\end{array}$} & \multirow[t]{2}{*}{$\begin{array}{l}\text { Portion out of all } \\
\text { sleep episodes }\end{array}$} & \multicolumn{3}{|c|}{$\begin{array}{l}\text { Onset of sleep } \quad \text { End of sleep } \\
\text { [hours before }(-) \text { or after }(+) \text { the closest tem- } \\
\text { perature minimum] }\end{array}$} & \multirow[t]{2}{*}{$\begin{array}{l}\text { Duration of } \\
\text { sleep (h) }\end{array}$} \\
\hline & & Range & Mean \pm Stan & d.-Dev. & \\
\hline $\mathbf{A}$ & $48 \%$ & -10 to -3.5 & $-6.70 \pm 1.88$ & $+3.68 \pm 1.90$ & $10.38 \pm 2.24$ \\
\hline B & $35 \%$ & -3 to +2 & $-0.98 \pm 1.09$ & $+5.98 \pm 1.53$ & $6.96 \pm 1.76$ \\
\hline $\mathrm{C}$ & $6 \%$ & +3 to +6 & $+4.8 \pm 1.7$ & $+10.3 \pm 1.6$ & $5.5 \pm 1.7$ \\
\hline D & $11 \%$ & +8 to +16 & $+11.5 \pm 2.3$ & $+16.4 \pm 2.3$ & $4.9 \pm 1.0$ \\
\hline
\end{tabular}


Effects of sleep on rectal temperature during internal desynchronization

$D$

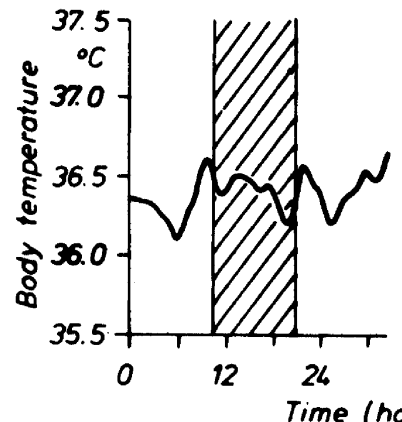

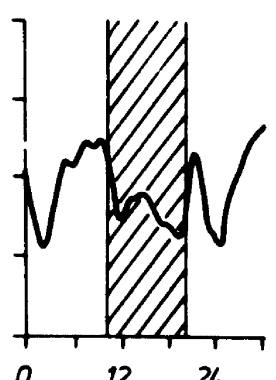

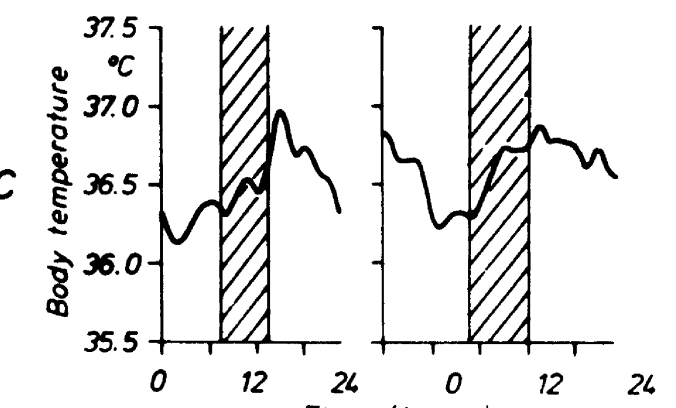

Four phase positions

of sleep within

the temperatur cycle:

$\boldsymbol{A}$

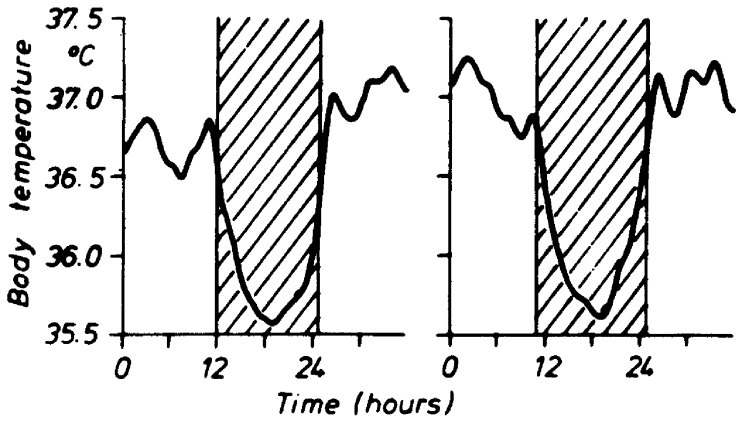

\section{$B$}

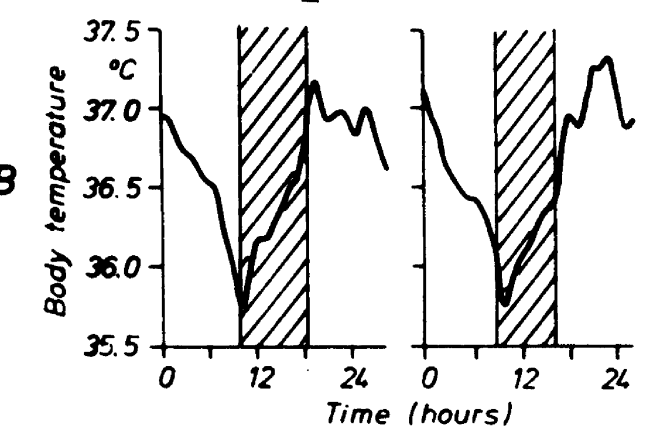

Fig. 4. Two examples from each of the four groups according to Fig. 3, presenting the course of rectal temperature around a sleep episode

in the cycle of deep body temperature, it accumulates, in the state of internal desynchronization, at several preferential positions.

Apart from phase of the rhythm of rectal temperature also the range of this rhythm is of interest. This parameter is recognizable from Fig. 4 where examples of temperature cycles in relation to the sleep-wake cycle are presented; from each of the preferred phase relationships (A to D in Fig. 3) two cycles are drawn originating from the same subject (with polygraphic sleep recordings) as shown in Fig. 1. Figure 4 demonstrates that the wave form and the range of the rectal temperature rhythm are influenced to a considerable amount by its temporal relationship to the sleep-wake cycle: during the sleep episodes, rectal temperature is always reduced, an effect which has been described as "masking effect" of activity on temperature (Aschoff 1970, Wever 1982). As a consequence, the temperature amplitude is large when sleep coincides with a temperature minimum (A), and it is small (or obscured) when sleep coincides with a temperature maximum (D).

A summarizing analysis of the relation between range of the temperature rhythm and the internal phase relationship from all ten subjects is given in Fig. 5. It shows a correlation between the two parameters; in particular, it shows that the range of the temperature rhythm is largest when sleep onset occurs shortly before the temperature minimum, and it is smallest when sleep onset occurs around the temperature maximum. Consequently, Fig. 5 demonstrates the influence of the sleep-wake rhythm on the rhythm of rectal temperature. 
Freerunning rhythm: internal desynchronization

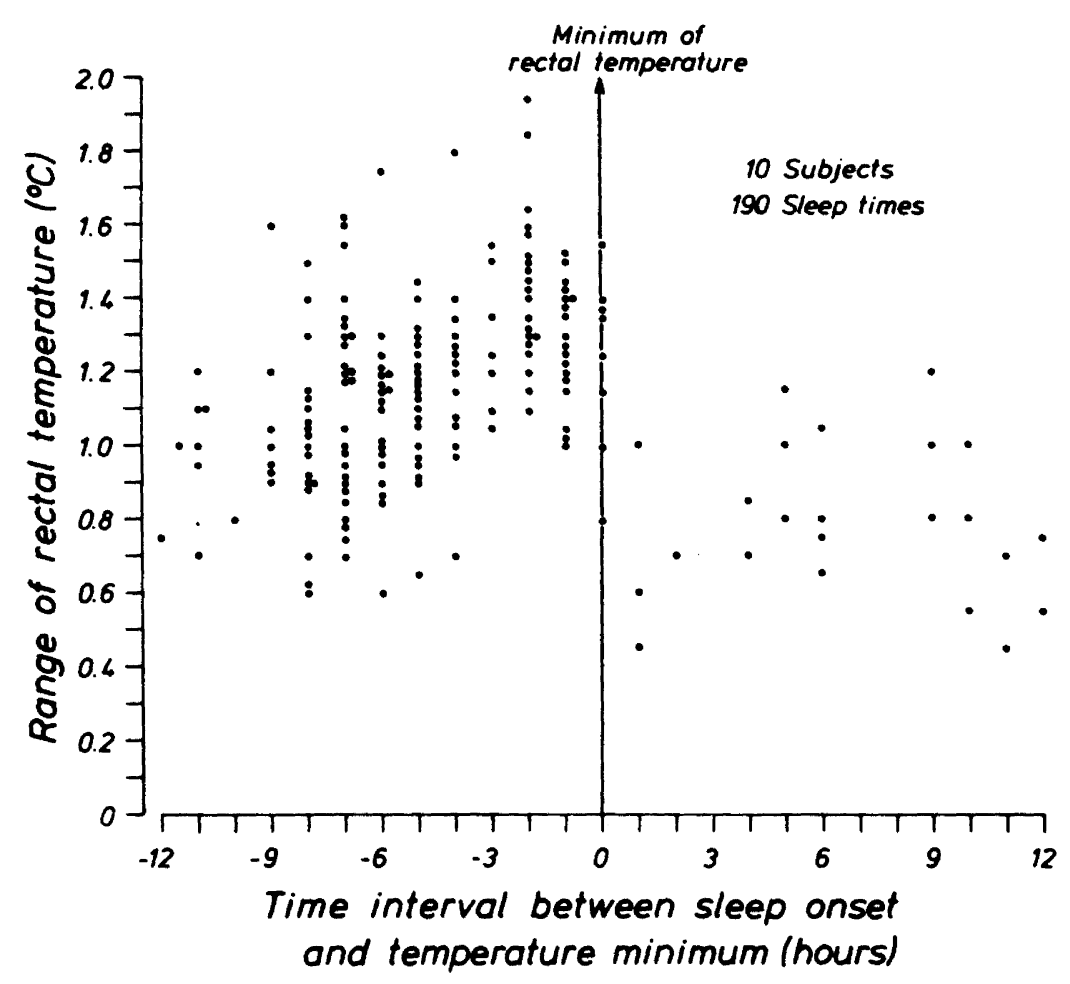

Fig. 5. Range of rectal temperature correlated to the phase position of sleep onset within the temperature cycle (reference: actual minimum of rectal temperature)

\section{Discussion}

The results obtained in experiments with internally desynchronized circadian rhythms show, on the one hand, a dependency of the sleep episodes on the rhythm of deep body temperature. The probability of the occurrence of a sleep episode varies with the phase of the temperature rhythm, and the duration of a sleep episode depends on its temporal position within the temperature cycle (cf. Fig. 3). On the other hand, the results also show a dependency of the rhythm of deep body temperature on the sleep-wake cycle. The range of the temperature rhythm depends on the phase relationship betwen the rhythms of temperature and sleep-wake (Fig. 5).

These significant results lead to the conclusion that there is mutual interaction between the circadian rhythms of activity and deep body temperature. This interaction is bidirectional and is present in spite of the missing mutual synchronization. As long as the rhythms run internally synchronized, only the mutual synchronization proves unambiguously mutual interaction; however, it cannot be differentiated whether this synchronization is due to an unidirectional or a bidirectional interaction. It is only from internally desynchronized rhythms that the additional conclusions to a bidirectional interaction can be drawn.

The mutual interaction between the two rhythms under consideration is based on, at least, three mechanisms which can be separated in the theoretical analysis.

1. The oscillatory interaction, which leads to internal synchronization inside and to internal relative coordination outside the mutual ranges of entrainment (Aschoff et al. 1967, Wever 1968). 
2. Beat phenomena, which are based on the collective and mutually superimposing contribution of two (or more) basic oscillators to every single overt rhythm (Wever 1979).

3. The masking effect, which is due to directly evoced responses of one overt rhythm to variations in the course of the other rhythm (see above).

In addition, the results demonstrates the clustering of the internal phase relationship between the rhythms of activity and rectal temperature in separated groups, representing preferred adjustment to some few discrete values. In internally synchronized rhythms, this phase relationship covers only a small range, corresponding to group B in Fig. 3 and Table 1 (Zulley et al. 1981). In internally desynchronized rhythms, in general, all phase relationships between $0^{\circ}$ and $360^{\circ}$ can occur. In particular, however, there is not an equal distribution of phase relationships, and not even a monomodal distribution. Instead, there are several preferred phase relationships between the two rhythms which are represented by the groups A through D (cf. Fig. 3 and Table 1). Apart from the two secondary groups $C$ and $D$, there is mainly group $A$ with the longest sleep durations which differs in its position from that of internally synchronized rhythms (these are characterized by a position similar to that of group B; Zulley et al. 1981). Phase relationships corresponding to the two groups $A$ and $B$ can also be observed in rhythms which are synchronized to an external zeitgeber with varying period. Under such an influence, the temporal relationship between sleep and minimum of rectal temperature does not change steadily, but jumps unsteadily between two discrete phase relationships, with the minimum near onset and near end of sleep (Wever 1981); these preferred phase relationships correspond to those in free-running internally desynchronized rhythms which are characterized by groups $A$ and $B$.

In all analyses discussed in this paper, actual values of single sleep episodes and single minima in rectal temperature are compared; this approach had been introduced by Zulley $(1976,1980)$ and Zulley and Schulz (1980). In addition, another type of analysis had been applied which compares single sleep episodes with the minimum of an educed shape of the temperature rhythm, i.e., with an average cycle of rectal temperature (Czeisler 1978, Czeisler et al. 1980a, 1980b). The results of this type of analysis deviate from results discussed in this paper in several details. For instance, a sleep episode begins preferably several hours before an actual minimum of rectal temperature (cf. Fig. 2, upper diagram) but several hours after the "educed" minimum (cf. Czeisler et al. 1980b). This difference is due to the systematic influence of sleep on the position of the temperature minima which results in systematic deviations between actual and "educed" minimum values. These deviations become obvious in Fig. 6 where the total course of rectal temperature (solid line) measured in an experiment with internally desynchronized rhythms (same experiment as in Fig. 1).is drawn together with a sinusoid which represents the educed rhythm of rectal temperature (constant period of $25.0 \mathrm{~h}$ ); in addition, the sleep episodes of the subject are indicated. As can be seen in Fig. 6 , the actual minimum temperature is mostly later than the educed minimum when it occurs near the onset of a sleep episode, and it is mostly earlier than the educed minimum when it occurs near the end of a sleep episode. This systematic shifting is another evidence for the influence of the sleep-wake rhythm on the temperature rhythm.

The variability of the rectal temperature rhythm in phase and amplitude could produce the impression of a random process. This impression, however, can be cor- 

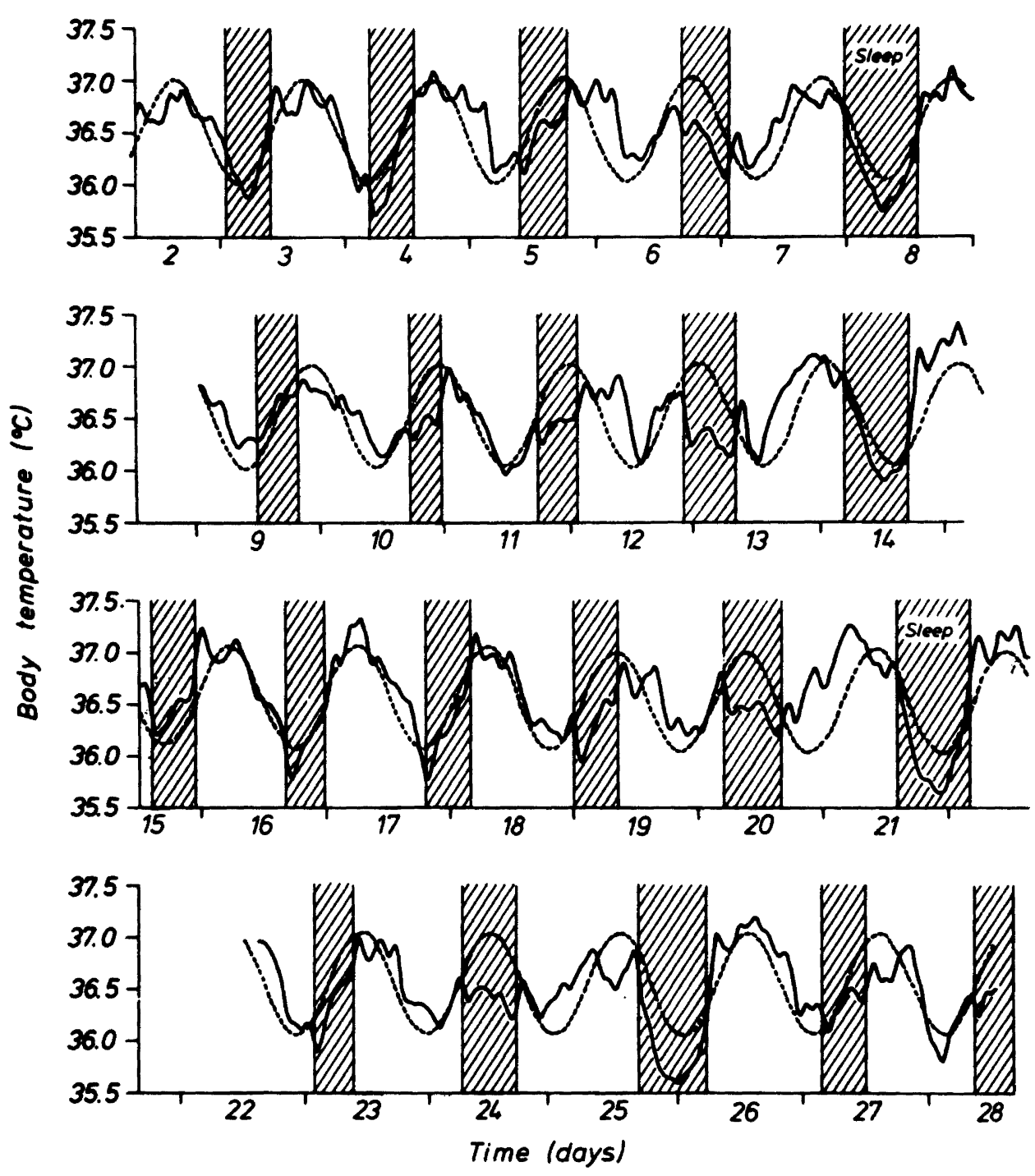

Fig. 6. Course of rectal temperature (solid line) measured in a 4-week experiment under constant conditions, presented in four consecutive sections (same experiment as in Fig. 1). Dotted line sine wave with constant parameters, best fitting the measured data. Sleep episodes are indicated by shaded areas

rected by the inspection of Fig. 6. In this figure, the dotted line (sinusoid) can be considered to represent the course of a hypothetical 'temperature oscillator' which runs with constant period and without any random fluctuations. As can be seen, the measured temperature values (constituting the overt temperature rhythm) fluctuate around the hypothetical oscillator course; for instance, the actual temperature minima are sometimes earlier and sometimes later than the hypothetical minima but return always to the values of the hypothetical oscillator. The deviations between actual and hypothetical temperature values depend, apart from random fluctuations, regularly on the temporal relation to the sleep episodes. This picture is hardly compatible with the assumption of a random generation of the temperature rhythm; in this case, the deviations from a long-term average should increase cumulatively. The picture of Fig. 6 is compatible with the assumption that the overt temperature rhythm is, in fact, influenced by the alteration between sleep and wake, but mainly controlled by an oscillator which must not necessarily interact with the sleep-wake rhythm. 
The results discussed in this paper are of theoretical interest because they give insight into the structure of the circadian multi-oscillator system. In addition, however, they are of practical interest because they enable to predict the sleeping behavior of a subject from the previously measured course of deep body temperature. In particular, they give the possibility to predict the duration of a sleep episode when the actual position of the temperature minimum is known. This holds true even during the state of internal desynchronization where the variations in sleep duration are remarkably large. On the other hand, the range of the temperature rhythm can be predicted from the phase relationship between sleep-wake and the temperature rhythm; in particular, this means the actual value of a temperature minimum can be predicted.

\section{References}

Aschoff J (1970) Circadian rhythms of activity and of body temperature. In: Hardy JD, Gagge AP, Stolwijk JA (eds) Physiological and behavioral temperature regulation. CC Thomas, Springfield, III, pp 905-919

Aschoff J, Gerecke U, Wever R (1967) Desynchronization of human circadian rhythms. Jpn J Physiol 17: 450-457

Czeisler CA (1978) Human circadian physiology: internal organization of temperature, sleep-wake, and neuroendocrine rhythms monitored in an environment free of time cues. $\mathrm{Ph} \mathrm{D}$ thesis, Stanford Univ

Czeisler CA, Weitzman ED, Moore-Ede MC, Zimmerman JC, Knauer RS (1980a) Human sleep: its duration and organization depend on its circadian phase. Science 210: 1264-1267

Czeisler CA, Zimmerman JC, Ronda JM, Moore-Ede MC, Weitzman ED (1980b) Timing of REM sleep is coupled to the circadian rhythm of body temperature in man. Sleep 2: 329-346

Wever RA (1968) Einfluss schwacher elektro-magnetischer Felder auf die circadiane Periodik des Menschen. Naturwissenschaften 55: 29-32

Wever RA (1975) The circadian multi-oscillator system of man. Int J Chronobiol 3: 19-55

Wever RA (1979) The circadian system of man. Springer, Berlin Heidelberg New York

Wever RA (1981) On varying work-sleep schedules: the biological rhythm perspective. In: Johnson LC, Tepas DI, Colquhoun WP, Colligan MJ (eds) Biological rhythms, sleep and shiftwork. Advances in sleep research, vol VII. Spectrum Publ. New York, pp 35-60

Wever RA (1982) Organization of the human circadian system: internal interactions. In: Wehr IA, Goodwin FK (eds) Circadian rhythms in psychiatry. Neuroscience Series. Boxwood Press, Los Angeles (in press)

Zulley J (1976) Schlaf und Temperatur unter freilaufenden Bedingungen. Berichte 30. Kongr Dtsch Ges Psychol. Hogrefe, Göttingen, pp 398-399

Zulley J (1979) Der Einfluss von Zeitgebern auf den Schlaf des Menschen. RG Fischer-Verlag, Frankfurt aM

Zulley J (1980) Duration and frequency of bedrest episodes in internally desynchronized rhythms. Sleep 2: 344

Zulley J, Schulz H (1980) Sleep and body temperature in freerunning sleep-wake cycles. In: Sleep 1978. 4th Eur Congr Sleep Res, Tirgu Mures, Karger, Basel, pp 341-344

Zulley J, Wever R, Aschoff J (1981) The dependence of onset and duration of sleep on the circadian rhythm of rectal temperature. Pfluegers Arch 391: 314-318 